\title{
Midinfrared electroluminescence in quantum cascade structures with InP/InGaAs active regions
}

\section{Citation}

Troccoli, Mariano, Federico Capasso, Jianxin Chen, Milton L. Peabody, Claire Gmachl, Deborah L. Sivco, Cheng-Hsuan Chen, and Alfred Y. Cho. 2003. "Midinfrared Electroluminescence in Quantum Cascade Structures with InP/InGaAs Active Regions." Journal of Applied Physics 94 (11): 7101-4. https://doi.org/10.1063/1.1625090.

\section{Permanent link}

http://nrs.harvard.edu/urn-3:HUL.InstRepos:41371359

\section{Terms of Use}

This article was downloaded from Harvard University's DASH repository, and is made available under the terms and conditions applicable to Other Posted Material, as set forth at http:// nrs.harvard.edu/urn-3:HUL.InstRepos:dash.current.terms-of-use\#LAA

\section{Share Your Story}

The Harvard community has made this article openly available. Please share how this access benefits you. Submit a story.

Accessibility 


\title{
Midinfrared electroluminescence in quantum cascade structures with InP/InGaAs active regions
}

\author{
Mariano Troccolia) and Federico Capasso ${ }^{\text {b) }}$ \\ Division of Engineering and Applied Sciences, Harvard University, 310 Cruft Laboratory, 19 Oxford Street, \\ Cambridge, Massachusetts 02138 \\ Jianxin Chen, Milton L. Peabody, Claire Gmachl, Deborah L. Sivco, Cheng-Hsuan Chen, \\ and Alfred Y. Cho \\ Bell Laboratories, Lucent Technologies, 600 Mountain Avenue, Murray Hill, New Jersey 07974
}

(Received 11 July 2003; accepted 19 September 2003)

\begin{abstract}
We report on the midinfrared emission from electroluminescent devices with quantum cascade active regions based on InGaAs/InP heterostructures. We observe emission at $\lambda \sim 12 \mu \mathrm{m}$ from two different structures and compare their emission characteristics based on the different band structure designs. Their relevance in view of the realization of InP-based quantum cascade lasers with aluminum-free waveguides is discussed. (C) 2003 American Institute of Physics.
\end{abstract}

[DOI: $10.1063 / 1.1625090]$

\section{INTRODUCTION}

The quantum cascade $(\mathrm{QC})$ laser $^{1}$ has been a breakthrough in the field of midinfrared (IR) sources that became feasible after the invention and development of growth techniques as sophisticated as molecular beam epitaxy (MBE), which allow the control of material properties down to the nanometer scale. One of the peculiarities of QC lasers, which makes them considerably different from conventional semiconductor lasers, is their unipolar nature, i.e., their operation relies entirely on one type of carrier (usually, electrons) undergoing intersubband transitions between levels created in one band by quantum confinement. This is achieved by alternately growing two semiconductor materials with different band gaps and exploiting the band offset between the two to create confined energy levels. The choice of layer thickness thus allows one to determine the active region characteristics, namely, its emission wavelength, the oscillator strength of the radiative transitions, and the electronic lifetimes of the energy levels. These characteristics can be tailored specifically to meet the user requirements, almost independently of the heterostructure material chosen for its realization, as long as the quantum wells are deep enough to allow for a large range of wavelengths. Up to now, laser action has been reported in a number of material systems, GaInAs/AlInAs, both lattice matched with InP (Ref. 2) and strained, ${ }^{3}$ and GaAs/AlGaAs with different $\mathrm{Al}$ mole fractions. ${ }^{4,5}$ The observation of intersubband electroluminescence in QC structures has also been reported for $\mathrm{Si} / \mathrm{SiGe}$ multilayers. ${ }^{6}$ Emission at wavelengths ranging from 3.5 to $93 \mu \mathrm{m}$ has been achieved. ${ }^{7,8}$

Here, we present the realization of various $\mathrm{InGaAs} / \mathrm{InP}$ quantum cascade electroluminescent devices emitting at a wavelength $\lambda \sim 12 \mu \mathrm{m}$. The importance of this semiconductor heterostructure, with respect to the usual QC lasers re-

\footnotetext{
a)Electronic mail: troccoli@ deas.harvard.edu

${ }^{\text {b) }}$ Electronic mail: capasso@deas.harvard.edu
}

sides in the capability of building a completely Al-free laser both in the waveguide claddings and in the active core. This may lead to improved thermal performances due to the substitution of a high thermal resistivity material (AlInAs) with a much lower one (InP), ${ }^{9}$ even though the presence of several interfaces in the QC active region might still play a significant role in the heat dissipation. An improvement in the thermal conductivity could, in any case, provide a considerable advantage in the development of QC laser technology, since these devices could reach room temperature continuous wave operation only recently, ${ }^{10}$ and there are still wide possibilities for further improvements.

One of the peculiarities of the InGaAs/InP heterostructure is the comparatively small band offset, which amounts to about half the value of the one in the lattice matched InGaAs/InAlAs material system. This may lead to higher leakage currents due to the tunneling of the injected electrons into the continuum of the conduction band. The advantages of this material system may be, therefore, more evident at longer wavelengths.

\section{SAMPLES}

The investigated samples included an active core consisting of five repetitions of an InGaAs/InP multilayer structure, grown on low $n$-doped $\left(n \sim 2 \times 10^{17} \mathrm{~cm}^{-3}\right)$ InP substrates. A highly doped $\left(n=10^{18} \mathrm{~cm}^{-3}\right)$ GaInAs contact layer was grown on top of the active core of each structure. The material growth was conducted in a MBE system with valved solid phosphorus and arsenic sources. Sample A had an active region designed as a so-called "bound-to-continuum"11 structure (Fig. 1), with one confined state used as the upper level of the radiative transition and the lower energy level of the radiative transition as part of a manifold of closely spaced states, a "miniband", which ensures rapid depletion of level 2 by electron-phonon scattering with small momentum transfer. The miniband states have also the purpose of electron injection into the active 


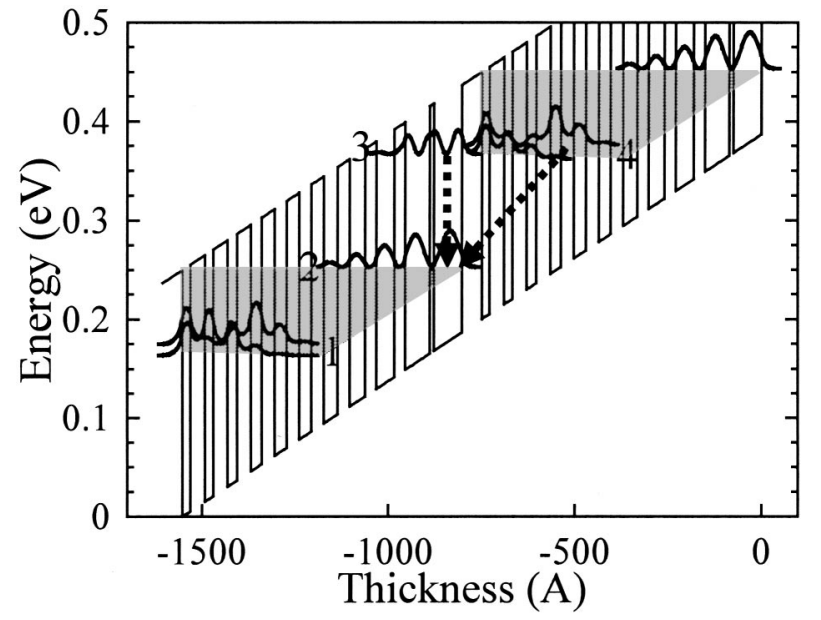

FIG. 1. Conduction band diagram of two periods of the active region and injector of sample A. Shaded areas indicate minibands of states. Arrows indicate the radiative transitions observed in the spectra. The layer sequence of one active region period (starting from the tunneling barrier, leftmost layer in figure) is: 5/2/4/2.2/3.8/2.6/3.7/2.9/3.5/3.2/3.4/3.3/3.3/3.7/3.2/4.1/ 3.1/4.8/3/6.5/1.1/7.5, where the dimensions are in $\mathrm{nm}$, InP layers are indicated in boldface type, and the underlined layers are the ones doped to $n$ $=2 \times 10^{17} \mathrm{~cm}^{-3}$.

region of the following period. The second sample (sample B) was designed using a "diagonal transition" scheme (Fig. 2). This last design should decrease the oscillator strength of the radiative transition but allow for a better confinement of the upper level, therefore increasing the excited state lifetime and limiting the thermally activated current leakage from the injector states into the conduction band continuum. We compared the two active regions in order to use them as the active core of QC laser waveguides.

The band structure of sample A was designed for emission at $12.2 \mu \mathrm{m}$. In Fig. 1, two periods of the active region conduction band structure are shown. The applied bias in this case, $V=0.2 \mathrm{~V}$ per stage, corresponds to the alignment of the

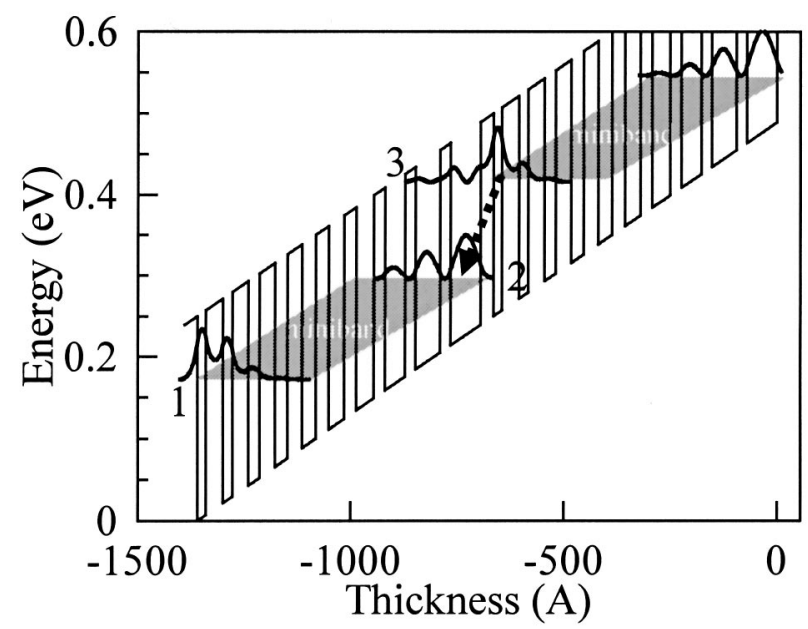

FIG. 2. Conduction band diagram of sample B. Two periods of active regions are shown. Shaded areas indicate the minibands. The layer sequence (starting from the tunneling barrier, leftmost layer in figure) is: $\mathbf{3} / 2 / \mathbf{4} / 2.2 /$ 3.8/2.6/3.7/2.9/3.4/3.2/3.1/3.6/2.8/4.2/2.6/4.7/2.5/5.7/2.5/7, where the dimensions are in $\mathrm{nm}$, InP layers are indicated in boldface type, and the underlined layers are the ones doped to $n=2 \times 10^{17} \mathrm{~cm}^{-3}$. (a)

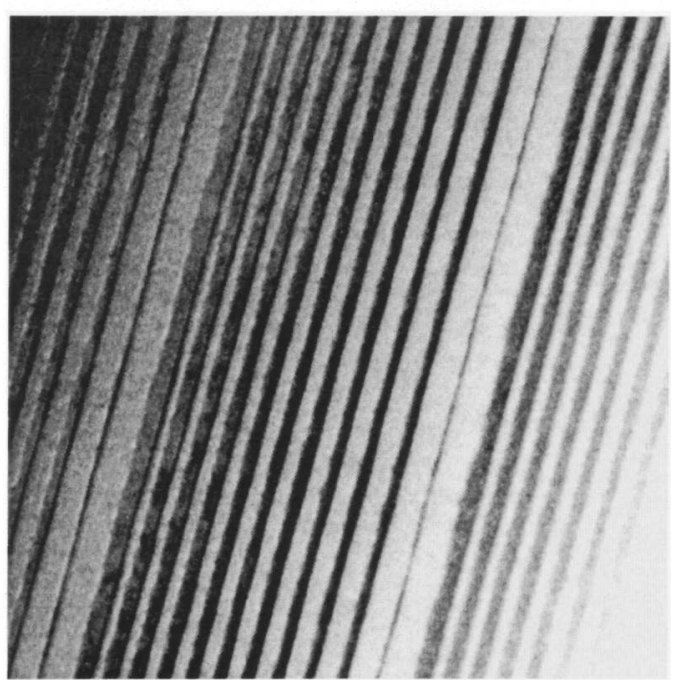

(b)

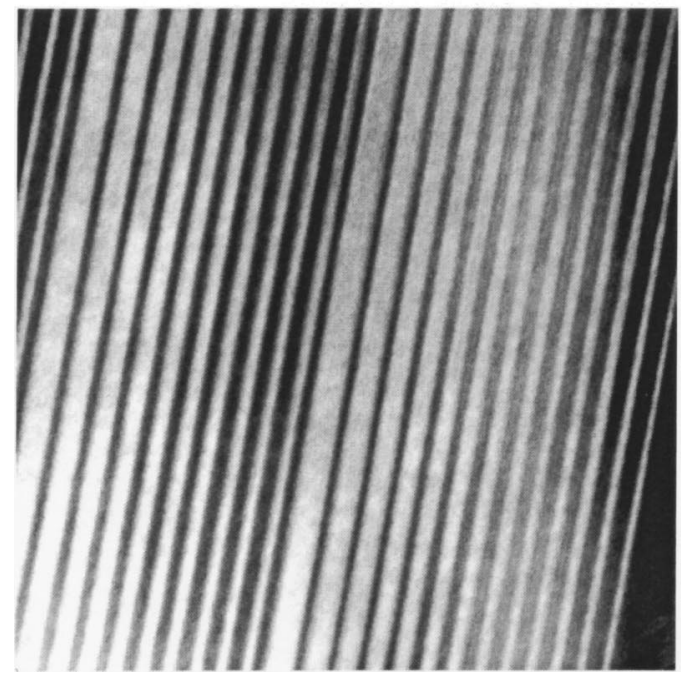

FIG. 3. Transmission electron microscope images of the two investigated samples: sample A (a) and sample B (b). Magnification is $10^{6} \times$. Dark layers are InP barriers and light gray layers are GaInAs wells.

upper state of the radiative transition with the ground state of the injector (state 4). In the usual cascading scheme, electrons are resonantly injected from the injection miniband into level 3, allowing for the radiative transition from level 3 to level 2 to take place. Figure 2 shows the band structure of sample $\mathrm{B}$, at a voltage of $V=0.25 \mathrm{~V}$ per stage. In this case, the emission wavelength is much more sensitive to the applied field due to the Stark shift of the radiative transition. At the bias corresponding to the band structure shown in Fig. 2, the wavelength of the intersubband emission is calculated to be at $10.5 \mu \mathrm{m}$.

The transmission electron microscope images of the multilayer part of the samples are shown in Figs. 3(a) and 3(b). In both cases, the layer thickness is close to the designed one and the repeatability from one period to another is very good. The interface quality appears to be good, although the attainment of laser action in QC devices may 
require further improvements in order to minimize the interface scattering contribution to the excited state non-radiative lifetime and to optimize injection.

\section{RESULTS}

The samples were etched into round mesa structures and a $\mathrm{Ti}(30 \mathrm{~nm}) / \mathrm{Au}(300 \mathrm{~nm})$ top contact was evaporated on top of each one. A Ge(12 nm)/Au(27 nm) $/ \operatorname{Ag}(50 \mathrm{~nm}) / \operatorname{Au}(200$ $\mathrm{nm})$ back contact was evaporated at the substrate side. The samples were then indium bonded onto copper holders and mounted into a helium-flow cryostat. The temperature was monitored with a calibrated Si diode mounted on the back of the sample holder and positioned directly opposite to the sample. Electroluminescence spectra were recorded from each sample under various bias conditions. A Fourier transform infrared (FTIR) spectrometer equipped with a liquid $\mathrm{N}_{2}$ cooled $\mathrm{HgCdTe}$ detector was used for the spectral measurements. In this configuration the FTIR was capable of detecting radiation up to $16 \mu \mathrm{m}$ with a resolution of $0.125 \mathrm{~cm}^{-1}$. Due to the long integration times required by the weak luminescence signals, the resolution was set at its lowest value, $32 \mathrm{~cm}^{-1}$. Electrical excitation to the samples was provided by a high-power HP8116A pulse generator, giving up to 100 $\mathrm{V}, 2$ A pulses. Typically, repetition rates around $80 \mathrm{kHz}$ were used and a combined step-scan and lock-in acquisition technique was employed to collect the spectra.

Figures 4(a) and 4(b) show the electroluminescence spectra of sample A recorded at $I=0.5$ and $1 \mathrm{~A}$, respectively, both with a duty cycle of $25 \%$ at a pulse repetition rate of $f_{\text {rep }}=84.3 \mathrm{kHz}$ and a temperature of $T=10 \mathrm{~K}$. The main peak at $815 \mathrm{~cm}^{-1}(12.2 \mu \mathrm{m})$ with a full width at half maximum of $70 \mathrm{~cm}^{-1}(8.7 \mathrm{meV})$ is clearly distinguishable in both spectra. When the current increases from $I=0.5 \mathrm{~A}$ up to $I=1 \mathrm{~A}$, another broader band at higher energies, which is already present at lower currents with a lower intensity, becomes clearly visible, rising to about $70 \%$ of the main peak intensity [Fig. 4(b)].

If we refer to the band structure, we can assign these two peaks to the transitions 3-2 and 4-2 in Fig. 1. As the drive current is increased, the ideal energy level alignment of Fig. 1 is lost. This reduces the injection efficiency into level 3, which is, therefore, occupied by a smaller fraction of the total electronic population. In addition, the electric field induced penetration of the wave functions in the barriers helps to increase the dipole of the radiative transition between the ground state of the injection miniband and state 2 (4-2 in Fig. 1). At this point, even though the radiative transitions from the injection miniband to the lower states of the following stage still have smaller oscillator strength than the "vertical" 3-2 transition, they become visible in the spectrum, with an intensity comparable to that of the main peak. The energies of the measured transitions (101 and $126 \mathrm{meV}$ ) in Fig. 3(b) closely correspond to the 3-2 and 4-2 transitions in the designed band structure (107 and $123 \mathrm{meV}$ ) at the corresponding bias. If we compare the spectra of sample A taken at different biases, corresponding to injected currents of $I$ $=0.3 \mathrm{~A}$ [not shown in Fig. 3(b), 0.5 and $1 \mathrm{~A}$, the high-energy peak corresponding to the 4-2 transition can be observed to
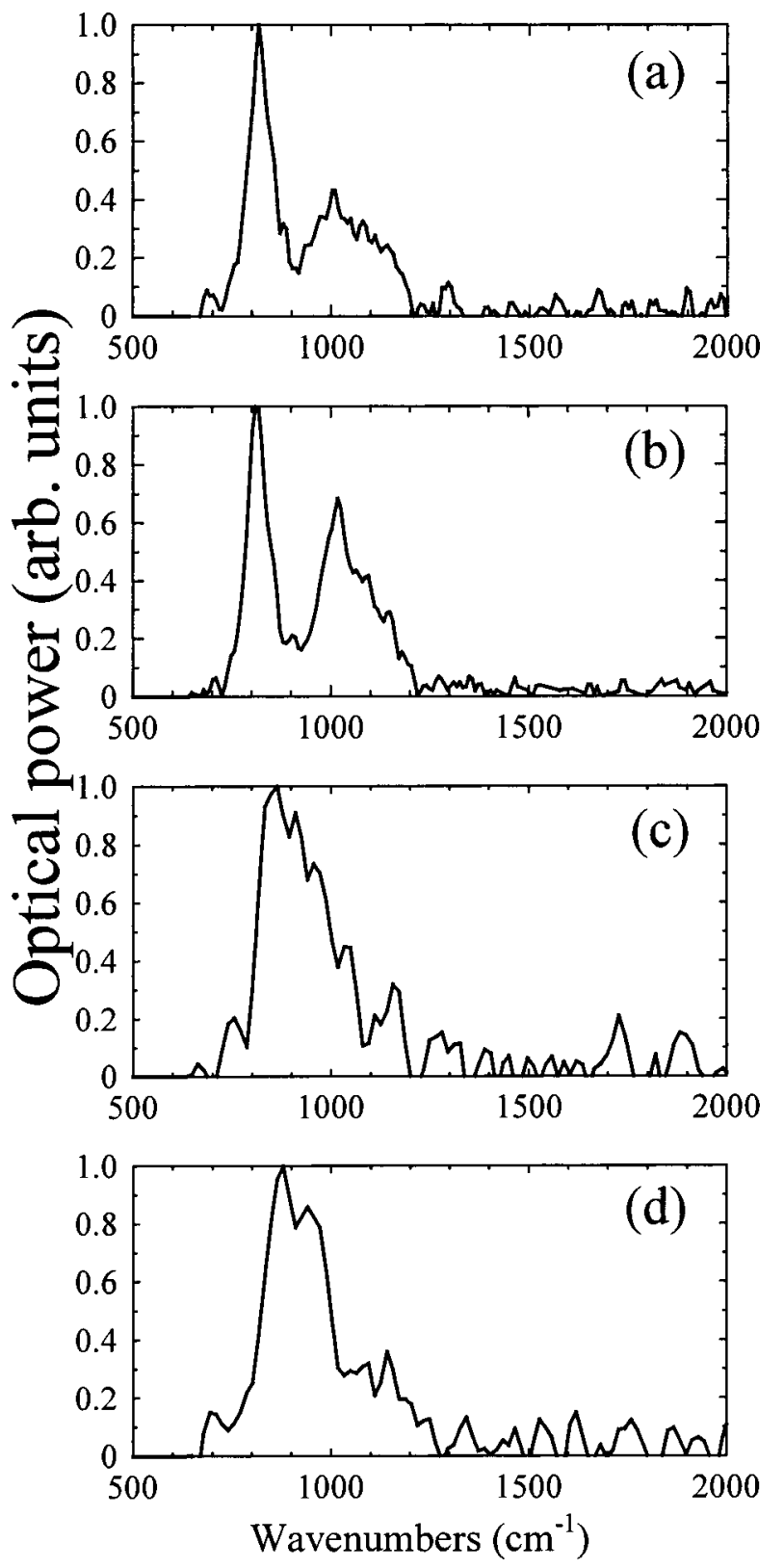

FIG. 4. Normalized spectra of sample A [(a) and (b)] and sample B [(c) and (d)] recorded at $T=10 \mathrm{~K}$ and current pulses of $I=0.5 \mathrm{~A}[(\mathrm{a})$ and (c)] and $I=1 \mathrm{~A}[(\mathrm{~b})$ and $(\mathrm{d})]$.

shift towards higher energies with increasing voltage, in agreement with the expected Stark shift. Moreover, comparing the spectra in Figs. 3(a) and 3(b) on a common scale and applying simple rate-equation considerations together with the calculation of the oscillator strengths of the radiative transitions, it is possible to estimate that the fraction of the injected electrons that populate the upper level of the optical transition (level 3 in Fig. 1) drops by about 35\% when passing from $I=500 \mathrm{~mA}$ to $I=1 \mathrm{~A}$, as a consequence of the reduced injection efficiency.

To avoid these problems related to the poor confinement of the upper energy level of the optical transition, we designed structure B, with a so-called diagonal transition between minibands (Fig. 2). Electronic relaxation by emission of optical phonons still allows for the rapid depletion of the 


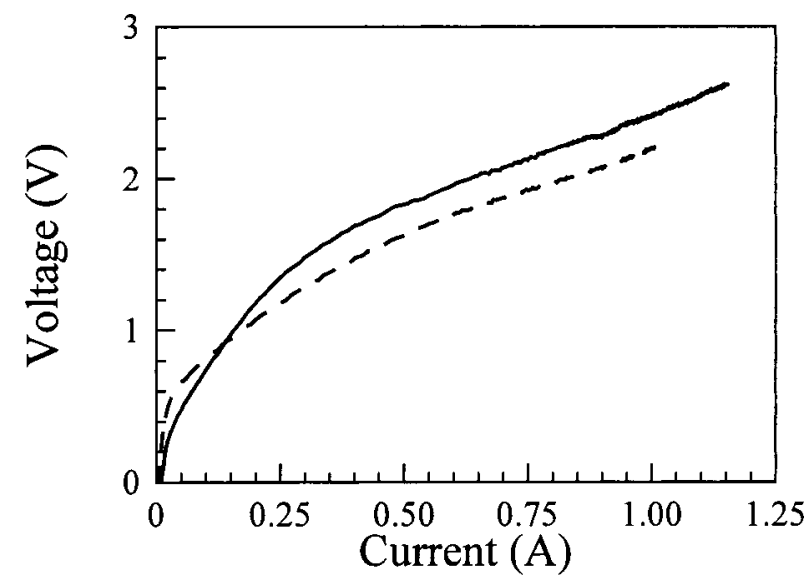

FIG. 5. Current-voltage characteristics of sample A (vertical transition, dashed line) and sample B (diagonal transition, solid line) measured at $T$ $=10 \mathrm{~K}$ with current pulses of $100 \mathrm{~ns}$ at $5 \mathrm{kHz}$ repetition rate.

lower level of the radiative transition (state 2 in Fig. 2) and at the same time contributes to the population of the upper level of the radiative transition in the following stage (ground state of the miniband-state 1). The periodic repetition of this structure allows for the usual cascading scheme to take place. In this case, the confinement of electrons in state 3 should be more efficient than in the vertical transition scheme since the energy barrier from level 3 to the top of the conduction band offset can be increased. The spectra measured from sample B in the weak $(I=0.5 \mathrm{~A})$ and strong $(I=1 \mathrm{~A})$ injection regime are shown in Figs. 4(c) and 4(d), respectively. As expected, only a single peak is observed in both cases. The peak is broader though than what is typically measured from intrawell intersubband transitions, since interminiband transitions may result as a convolution of multiple intersubband ones. Its peak energy is higher than the 3-2 transition of sample A (109 vs $101 \mathrm{meV})$, and reasonably close to its predicted value $(113 \mathrm{meV})$. The broadness of the transition makes the tuning of the emission with the increase in the electric field inside the structure not clearly visible.

The current-voltage characteristics of the two samples are shown in Fig. 5. They have been measured in pulsed mode with a duty cycle of $0.05 \%$ to make sure that the sample temperature does not vary during the measurements. The current and voltage values were acquired by gated boxcar averagers and recorded by a HP4155 semiconductor pa- rameter analyzer. The typical QC behavior with a diode-like $I-V$ can be observed. The diagonal transition structure (sample B, continuous line) shows a more leaky $I-V$ at low current levels, possibly due to the smaller thickness of a full active region period, thus allowing thermally activated electrons to tunnel through more easily even before the voltage drop corresponding to the band alignment of Fig. 2 is reached. At high currents, in the regime of strong injection, the two differential resistances become very similar $\left(R_{d}\right.$ $=1 \Omega$ for sample A and $R_{d}=1.16 \Omega$ for sample $\mathrm{B}$ ), most probably because in both designs carrier leakage into the continuum starts playing an important role in this region of the transport characteristics.

\section{CONCLUSIONS}

In conclusion, we report the mid-IR emission from QC electroluminescent structures with $\mathrm{InGaAs} / \mathrm{InP}$ active regions. This material system may be useful for the realization of improved QC lasers. Some of the issues that are to be taken into account for the realization of a QC laser have been pointed out, and will be the object of future investigations.

\section{ACKNOWLEDGMENTS}

This work was partly supported by DARPA/US ARO under Contract No. DAAD19-00-C-0096.

${ }^{1}$ F. Capasso, C. Gmachl, D. L. Sivco, and A. Y. Cho, Phys. Today 55, 34 (2002); F. Capasso, R. Paiella, R. Martini, R. Colombelli, C. Gmachl, T. L. Myers, M. S. Taubman, R. M. Williams, C. G. Bethea, K. Unterrainer, H. Y. Hwang, D. L. Sivco, A. Y. Cho, A. M. Sergent, H. C. Liu, and E. A. Whittaker, IEEE J. Quantum Electron. 38, 511 (2002).

${ }^{2}$ J. Faist, F. Capasso, D. L. Sivco, C. Sirtori, A. L. Hutchinson, and A. Y. Cho, Science 264, 553 (1994).

${ }^{3}$ R. Köhler, C. Gmachl, A. Tredicucci, F. Capasso, D. L. Sivco, S. N. G. Chu, and A. Y. Cho, Appl. Phys. Lett. 76, 1092 (2000).

${ }^{4}$ C. Sirtori, P. Kruck, S. Barbieri, P. Collot, J. Nagle, M. Beck, J. Faist, and U. Oesterle, Appl. Phys. Lett. 73, 3486 (1998).

${ }^{5}$ C. Becker, C. Sirtori, H. Page, G. Glastre, V. Ortiz, X. Marcadet, M. Stellmacher, and J. Nagle, Appl. Phys. Lett. 77, 463 (2000).

${ }^{6}$ G. Dehlinger, L. Diehl, U. Gennser, H. Sigg, J. Faist, K. Ensslin, D. Grützmacher, and E. Müller, Science 290, 2277 (2000).

${ }^{7}$ J. Faist, F. Capasso, D. L. Sivco, A. L. Hutchinson, S. N. G. Chu, and A. Y. Cho, Appl. Phys. Lett. 72, 680 (1998).

${ }^{8}$ R. Köhler, A. Tredicucci, F. Beltram, H. E. Beere, E. H. Linfield, A. G. Davies, D. A. Ritchie, R. C. Iotti, and F. Rossi, Nature (London) 417, 156 (2002).

${ }^{9}$ C. Gmachl, A. Tredicucci, F. Capasso, A. L. Hutchinson, D. L. Sivco, J. N. Baillargeon, and A. Y. Cho, Appl. Phys. Lett. 72, 3130 (1998).

${ }^{10}$ M. Beck, D. Hofstetter, T. Aellen, J. Faist, U. Oesterle, M. Ilegems, E. Gini, and H. Melchior, Science 295, 301 (2002).

${ }^{11}$ J. Faist, M. Beck, T. Aellen, and E. Gini, Appl. Phys. Lett. 78, 147 (2001). 